\title{
El futuro de
}

la administración

Trump: dos

escenarios

en prospectiva*

/ The future of

the Trump

administration:

two scenarios in

prospective

* Artículo de investigación científica. Recibido: 26 de abril de 2017. Aceptado: 7 de junio de 2017. Tla-Melaua, revista de Ciencias Sociales. Facultad de Derecho y Ciencias Sociales. Benemérita Universidad Autónoma de Puebla, México / IssN: 1870-6916 / Nueva Época, año 12, núm. 44, abril-septiembre 2018, pp. 120-140. 
José Antonio Cisneros Tirado*

Melva Guadalupe Navarro Sequeira**

Renee Scherlen***

RESUMEN

Donald Trump llevó a cabo una muy polémica campaña política. Muchos de sus mensajes pueden considerarse xenófobos, misóginos y racistas. Al final del primer año de su presidencia, Trump ha firmado más decretos presidenciales (executive orders) que cualquier otro presidente durante este mismo lapso. Estos decretos han confrontado directamente algunas de las acciones más prominentes de la anterior administración, lo cual ha contribuido a modificar significativamente el panorama político en los Estados Unidos. Desde su inicio, esta administración ha estado plagada de escándalos. Destaca la investigación federal que actualmente se conduce sobre la posible colusión entre la campaña política de Trump y el gobierno ruso. En esta investigación se presentan dos escenarios prospectivos sobre el futuro del actual presidente de los Estados Unidos, uno con base en la teoría de responsabilidad política (political accountability) y el otro desarrollado sobre el voto económico.

\section{PALABRAS CLAVE}

Donald Trump, prospectiva política, voto económico, responsabilidad política, impugnación presidencial.
A B S TRACT

Donald Trump carried out a very controversial political campaign. Many of his messages can be considered xenophobic, misogynistic and racist. At the end of the first year of his presidency, Trump has signed more executive orders than any other president during this same period. These decrees have directly confronted some of the most prominent actions of the previous administration that has significantly change the political scene in the United States. From the start, this administration has been plagued by scandals. The federal investigation that is currently conducted on the possible collusion between the political campaign of Trump and the Russian government is a good example. This research paper presents two scenarios about the future of the current president of the United States, one based on the theory of political responsibility (political accountability) and the other developed on the economic vote.

KEYWORDS

Donald Trump, political prospective, economic vote, political responsibility, presidential impeachment.

\footnotetext{
* Profesor investigador en el Instituto de Ciencias de Gobierno y Desarrollo Estratégico de la Benemérita Universidad Autónoma de Puebla, México. (joseantonio.cisneros@correo.buap.mx) orcid.org/00000002-8825-5467

** Profesora investigadora en el Instituto de Ciencias de Gobierno y Desarrollo Estratégico de la Benemérita Universidad Autónoma de Puebla, México. (melva.navarro@correo.buap.mx) orcid.org/0000-00023302-1989

*** Profesor titular de Ciencia Política del Departamento de Estudios de Justicia y Gobierno de la Appalachian State University, Estados Unidos. (scherlenrg@appstate.edu) orcid.org/0000-0002-0475-290X
} 
1. Introducción / 2. Impugnaciones de presidentes en Estados Unidos / 3. Los decretos ejecutivos del presidente Trump / 4. Responsabilidad política, voto económico y construcción de escenarios políticos / 5. Relación entre aceptación presidencial y crecimiento económico / 6. Relación entre estabilidad política y desempeño presidencial / 7. Conclusiones / 8. Bibliografía

\section{INTRODUCCIÓN}

El desarrollo de escenarios consiste en construir futuros factibles sobre contextos específicos, con base en supuestos teóricos y condiciones. Estos estudios requieren que un fenómeno se estudie desde un punto de vista retrospectivo, identificando actores, hechos o grupos sociales que pueden influir en la construcción de diversos escenarios. ${ }^{1}$

Esta investigación no propone una lista exhaustiva de escenarios posibles, sino dos futuros realizables para la presente administración. El primer futuro tiene como base la teoría de voto económico. Se toman en cuenta los estudios previos sobre el tema, respecto a la correlación entre el desempeño general de la economía y la reelección de los representantes. De tal forma, se presenta un escenario que relaciona la aceptación y popularidad de Trump contra el desempeño económico de su gobierno, para determinar si el actual presidente y su partido lograrán una reelección, tanto en el las elecciones intermedias de 2018, como en las presidenciales del 2020. ${ }^{2}$

El segundo escenario, sustentado en la teoría de responsabilidad política, correlaciona la estabilidad en el ambiente político, contra una evaluación del desempeño de la figura presidencial. En este futuro, también se evalúa una matriz de resultados respecto a la reelección, tanto de Trump, como del Partido Republicano en los próximos comicios. ${ }^{3}$ Como se mencionó anteriormente, este trabajo no pretenden hacer una presentación exhaustiva de los escenarios posibles, sino de dos de ellos cuya ocurrencia es factible, considerando supuestos específicos para cada uno. ${ }^{4}$

\footnotetext{
${ }^{1}$ Mahmoud, Mohammed, Liu, Yuqiong, Hartmann, Holly, Stewart, Steven, Wagener, Thorsten, Semmens, Darius, et al., "A formal framework for scenario development in support of environmental decisionmaking”, Environmental Modelling \&̊ Software, vol. 24, núm. 7.

${ }^{2}$ Lewis-Beck, Michael, \& Nadeau, Richard, "Economic voting theory: Testing new dimensions", Electoral Studies, vol. 30.

${ }^{3}$ Nardulli, Peter, Popular Efficacy in the Democratic Era: A Reexamination of Electoral Accountability in the United States, 1828-2000, Princeton, Princeton University Press, 2005.

${ }^{4}$ Cely, Alexandra, "Metodología de los Escenarios para Estudios Prospectivos", Revista Ingeniería e Investigación, núm. 44, pp. 26-35.
} 
Este trabajo se compone de las siguientes partes. En primer lugar, a manera de marco contextual, se revisan tanto la historia de la impugnación presidencial en Estados Unidos, como los decretos ejecutivos más controversiales emitidos por el presidente Trump durante su primer año en el poder. A continuación, se muestra una revisión de las teorías de responsabilidad política, voto económico y desarrollo de escenarios futuros. Finalmente, se presentan dos matrices de prospectiva con su respectivo análisis, conclusiones y limitaciones.

\section{ImPugnaciones DE PRESIDENTES EN ESTADOS UNidOS}

La Constitución de los Estados Unidos establece los principios que rigen los aspectos procedimentales de una impugnación presidencial. La Cámara de Representantes tiene el poder para llevar a cabo una impugnación y el Senado verifica el proceso de juicio político después de que los artículos de impugnación (articles of impeachment) son aprobados al interior del congreso. Ambas consideraciones se establecen en el primer artículo constitucional.

La Suprema Corte también juega un papel dentro de estos procedimientos, dado que el magistrado presidente de dicho tribunal dirige este proceso. El presidente no puede ser impugnado sin la aprobación de dos terceras partes del Senado. En caso de ser encontrado culpable, será sujeto de acusación, juicio, procesamiento y castigo de acuerdo a derecho. El presidente podrá ser impugnado únicamente en caso de traición, soborno, así como otros delitos o causas graves. ${ }^{5}$

A lo largo de la historia estadounidense, solamente dos presidentes han sido impugnados, pero ninguno fue condenado por dichos cargos. El primer caso fue el del presidente Andrew Johnson, el décimo séptimo presidente de los Estados Unidos. Fue acusado de violar la Ley de Duración del Mandato (Tenure of Office Act) de 1867. Este código establecía que el Senado debería aprobar la remoción de un miembro del gabinete si este había contado con la aprobación de la cámara alta del congreso. La Cámara de Representantes impugnó a Andrew Johnson después de que dicho presidente removiera del cargo a su secretario de Guerra, el republicano Edwin Stanton. Sin embargo, el Senado absolvió a Johnson dado que faltó solamente un voto a favor de condenar criminalmente al presidente. ${ }^{6}$

William Jefferson Clinton fue el cuadragésimo segundo presidente de los Estados Unidos. Fue impugnado por la Cámara de Representantes en 1998 por mentir bajo juramento durante su audiencia ante un gran jurado

\footnotetext{
5 "An Overview of the Impeachment Process", United States Senate. [Consulta: 16 de diciembre, 2017]. Disponible en: https://www.senate.gov/reference/resources/pdf/98-806.pdf

6 "List of presidents who were impeached", ThoughtCo, lifelong learning. [Consulta: 10 de enero, 2017]. Disponible en: https://www.thoughtco.com/presidents-who-were-impeached-3368130
} 
respecto a una relación extramarital con su asistente Monica Lewinsky al interior de la Casa Blanca. El presidente Clinton de igual forma persuadió a otras personas a mentir sobre el incidente. Los cargos presentados en su contra fueron perjurio y obstrucción de justicia.

Después de enfrentar un juicio, Clinton fue absuelto de ambos cargos y fue obligado a emitir una disculpa pública por sus hechos. A pesar de que pudo terminar su segundo periodo presidencial, la reputación de Clinton nunca pudo resarcirse. Esto obviamente impactó de forma negativa las aspiraciones presidenciales de su cónyuge, Hillary Clinton, durante su campaña en $2016 .^{7}$

Otro incidente de similar naturaleza se observa en el caso del presidente Richard Nixon, en 1974, quien llegó a estar muy cerca de una condena criminal como resultado del proceso de impugnación. Sin embargo, el trigésimo séptimo presidente renunció antes de enfrentar los cargos derivados del escándalo Watergate, al haber sido acusado de abusar de sus facultades presidenciales y usar los órganos federales para espiar a sus rivales políticos. En 1972, existió un allanamiento orquestado por Nixon en el cuartel general del Partido Demócrata. No obstante, el presidente trató de encubrir este acto, lo cual involucró hasta la Suprema Corte y una posible crisis constitucional.

Igual que en el caso Clinton, asistentes cercanos a Nixon estuvieron involucrados en espionaje e intervención telefónica en contra de enemigos políticos del presidente. Esta administración utilizó los recursos del Buró Federal de Investigación (FBI), la Agencia Central de Inteligencia (CIA), así como el departamento de hacienda (IRS) para investigar a ciertos activistas y rivales políticos. Después de que el Congreso norteamericano llevara a cabo una investigación, se encontraron evidencias relevantes que probaban el abuso de autoridad y facultades por parte de Nixon, por lo que los artículos de impugnación (Articles of Impeachment) fueron invocados en su contra. Richard Nixon renunció a la presidencia en 1974; su sucesor, Gerald Ford, le otorgó el perdón presidencial bajo una creciente presión política. ${ }^{8}$

A través de la historia de los Estados Unidos, únicamente se han invocado los artículos de impugnación en contra de un presidente en funciones; ninguno de estos casos concluyó en una condena en contra del ejecutivo. En resumen, han existido tres tipos de conducta que han conducido a un proceso legal de impugnación: el abuso de poder para fines personales,

\footnotetext{
7 "Impeachment inquiry: William Jefferson Clinton, President of the United States". U.S. Government Publishing Office. [Consulta: 10 de enero, 2017]. Disponible en: https://www.gpo.gov/fdsys/pkg/GPOCDOC-106sdoc3/pdf/GPO-CDOC-106sdoc3-9.pdf

8 "The Watergate Scandal", The Constitutional Rights Foundation. [Consulta: 11 de enero, 2017]. Disponible en: http://www.crf-usa.org/images/pdf/members/bria25_4wb.pdf
} 
conducta indecorosa, impropia de la investidura presidencial, así como la ejecución inadecuada de las funciones y propósitos del cargo. ${ }^{9}$

Todos y cada uno de estos actos han representado delitos graves, sin embargo, el hecho de que en ninguno de los casos presentados se haya llegado a una condena criminal demuestra que dentro de la política norteamericana existe un alto respeto y propósito de proteger la investidura presidencial. Lo anterior se debe a la relevancia simbólica que esta institución conlleva y lo que representa para el pueblo norteamericano. A continuación, se revisan los decretos presidenciales más controversiales de la actual administración.

\section{LOS DECRETOS EJECUTiVOS DEL PRESIDENTE TRUmP}

El cuadragésimo quinto presidente de los Estados Unidos ha firmado más decretos ejecutivos (executive orders) que ninguno de sus predecesores durante el primer año de su mandato. ${ }^{10}$ En sus primeros 100 días, Trump ya había firmado más de noventa decretos y memorándums presidenciales, muchos de ellos con la intención de abolir algunos de los actos más relevantes del presidente Obama. ${ }^{11}$ Un decreto ejecutivo le proporciona al presidente la facultad de asumir un rol legislativo desde esta rama del gobierno.

Históricamente, este recurso había sido usado por los presidentes norteamericanos como herramientas gerenciales para conducir e implementar las políticas de las agencias que pertenecen al gabinete sin requerir la aprobación directa del congreso. Estos mandatos permitían al presidente establecer códigos, procedimientos y delegar responsabilidades a las diversas agencias que forma parte del gobierno federal y que dependen del ejecutivo. No obstante, dichos decretos cuentan con una total validez jurídica y son implementadas de forma inmediata.

Observamos algunos ejemplos de la importancia que pueden tener estas órdenes presidenciales en la compra del estado de Luisiana, la anexión de Texas, el fin de la segregación racial en las fuerzas armadas o la creación de campos de concentración para estadounidenses nipones durante la Segunda Guerra Mundial. ${ }^{12}$

\footnotetext{
9 "Impeachment and Removal", Congressional Research Service. [Consulta: 13 de enero, 2017]. Disponible en: https://fas.org/sgp/crs/misc/R44260.pdf

10 "Trump is on pace to sign more executive orders than any president in the last 50 years", $C \mathcal{N N}$. [Consulta: 29 de agosto, 2017]. Disponible en: http://edition.cnn.com/2017/10/13/politics/donald-trumpexecutive-orders/index.html

11 "Trump signed 90 executive actions in his first 100 days", Business Insider. [Consulta: 4 de mayo, 2017]. Disponible en: http://www.businessinsider.com/trump-executive-orders-memorandum-proclamationspresidential-action-guide-2017-1

${ }^{12}$ Ginsberg, Benjamin, Lowi, Theodore., \& Weir, Margaret, We the People: An Introduction to American Politics, New York, W. W. Norton \& Company, 2014.
} 
Además de los decretos, el presidente Trump ha firmado más de treinta memorándums presidenciales, los cuales ni siquiera requieren publicarse de forma oficial, dado que delegan responsabilidades que recaen dentro de las facultades asignadas al poder ejecutivo. Asimismo, Trump ha emitido más de cien proclamas públicas con diversos motivos. Durante el mandato presidencial más largo en la historia de los Estados Unidos, liderado por Franklin Delano Roosevelt (1933-45), el presidente firmó más de 3700 decretos ejecutivos. No obstante, Roosevelt debió superar los efectos de la Gran Depresión y la entrada de los Estados Unidos a la Segunda Guerra Mundial.

Según Donald Trump, su administración está llevando a cabo una profunda reforma del gobierno, dado que considera haber heredado un completo desorden por parte de su predecesor. ${ }^{13}$ Durante los ocho años de la administración de Barack Obama se firmó un total de 277 decretos ejecutivos. Esto atrajo numerosas críticas por parte de la oposición, incluyendo a Donald Trump, acusando a Obama de "gobernar por decreto". ${ }^{14}$

Tabla 1. Decretos presidenciales de Donald Trump ${ }^{15}$

\begin{tabular}{|c|l|}
\hline $\begin{array}{c}\text { Decretos } \\
\text { presidenciales }\end{array}$ & \multicolumn{1}{c|}{ Contenido y alcances } \\
\hline $\begin{array}{c}\text { Este decreto establece que los padres fundadores buscaban incluir a las } \\
\text { "voces religiosas" en el debate de la esfera pública de la nación, por lo que } \\
\text { este mandato busca proteger la práctica de la fe sin temor a ser perseguido } \\
\text { o sancionado por parte del gobierno federal. Con ello, el Departamento } \\
\text { del Tesoro no podrá imponer ninguna sanción a cualquier organización } \\
\text { religiosa o ministro de fe que se pronuncie a favor o en contra de un } \\
\text { candidato a un cargo de elección popular basado sus creencias religiosas. } \\
\text { Libertad de Expresión } \\
\text { y la Libertad Religiosa } \\
\text { del } 4 \text { de mayo de 2017. }\end{array}$ & $\begin{array}{l}\text { De igual forma, ninguna agencia del Gabinete, ya sea la Secretaría del } \\
\text { Trabajo, del Tesoro o de Salud podrá imponer sanción alguna a cual- } \\
\text { empleados, especialmente mujeres, en relación con su salud reproductiva, } \\
\text { anticonceptivos y cuidado prenatal. Además de las implicaciones para } \\
\text { las mujeres y la posible discriminación hacia la comunidad LGBT en tér- } \\
\text { minos laborales o profesionales, este decreto atenta contra la separación } \\
\text { entre la Iglesia y el Estado. Contrario al texto de este mandato, Thomas } \\
\text { Jefferson, uno de los padres fundadores, expresó en 1802 que debería } \\
\text { existir "una muralla entre la Iglesia y el Estado. De forma posterior, en } \\
\text { 1954, se promulgó la Enmienda Johnson, la cual prohibía a cualquier } \\
\text { organización religiosa de participar en los procesos electorales, ya fuera } \\
\text { a favor o en contra de un candidato. }\end{array}$ \\
\hline
\end{tabular}

\footnotetext{
13 "Trump has already signed 66 executive actions; here is what each one does", Business Insider. [Consulta: 25 de mayo, 2017]. Disponible en: http://www.businessinsider.com/trump-executive-orders-memorandum-proclamations-presidential-action-guide-2017-1

14 "Candidate Trump attacked Obama's executive orders. President Trump loves executive orders", The Washington Post. [Consulta: 10 de noviembre, 2017]. Disponible en: https://www.washingtonpost.com/ news/monkey-cage/wp/2017/10/17/candidate-trump-attacked-obamas-executive-orders-presidenttrump-loves-executive-orders/?utm_term=.c12586dc46fb

${ }^{15}$ La traducción es de los autores.
} 


\begin{tabular}{|c|c|}
\hline $\begin{array}{l}\text { Decreto Presidencial } \\
\text { para Comprar } \\
\text { Productos Americanos } \\
\text { y Emplear } \\
\text { Americanos, del } 18 \text { de } \\
\text { abril de } 2017 .\end{array}$ & $\begin{array}{l}\text { Esta orden instruye a todas las agencias federales para evaluar y proponer } \\
\text { cambios sustanciales al programa de visas H-1B, el cual está diseñado } \\
\text { para atraer y emplear trabajadores especializados. Los críticos de este } \\
\text { programa consideran que su propósito es contratar mano de obra extran- } \\
\text { jera barata. No obstante, dicho programa ha sido implementado para } \\
\text { atraer trabajadores altamente capacitados para sectores que enfrentan } \\
\text { dificultades significativas para contratar estadounidenses. Esta medida } \\
\text { también dirige a las agencias federales para que maximicen su consumo } \\
\text { de productos americanos, tales como acero, hierro, aluminio y cemento. }\end{array}$ \\
\hline $\begin{array}{l}\text { Decreto presiden- } \\
\text { cial para Proteger } \\
\text { a la Nación de la } \\
\text { Entrada de Terroristas } \\
\text { Extranjeros a los } \\
\text { Estados Unidos del } 6 \\
\text { de marzo de } 2017 .\end{array}$ & $\begin{array}{l}\text { Como parte de sus promesas de campaña, Donald Trump hizo un com- } \\
\text { promiso con los estadounidenses sobre el aumento de la seguridad fron- } \\
\text { teriza, especialmente para limitar la migración de países musulmanes. La } \\
\text { prohibición de viajar (Travel Ban) fue establecida a finales de enero, pero } \\
\text { un juez federal la declaró como inconstitucional debido a que afectaba } \\
\text { espećficamente a los musulmanes, esto en violación de la libertad reli- } \\
\text { giosa establecida en la Primera Enmienda. Una segunda versión de esta } \\
\text { prohibición fue promulgada en marzo de } 2017 \text {. Algunos de sus objetivos } \\
\text { específicos son los siguientes: reducir drásticamente el número de refugia- } \\
\text { dos admitidos en los Estados Unidos para } 2017 \text { de } 110000 \text { a } 50 \text { 000; y } \\
\text { suspender la entrada a suelo norteamericano a personas provenientes de } \\
\text { Irán, Sudán, Libia, Yemen, Somalia y Siria durante } 90 \text { días. Esta orden } \\
\text { también suspendía el Programa de Exención de Entrevista para Visas, lo } \\
\text { cual evitaba que las personas realizaran este trámite de forma presencial } \\
\text { y, con ello, lo hacía más complicado para los inmigrantes. En esta ocasión, } \\
\text { jueces federales de Hawái y Maryland bloquearon esta nueva versión de } \\
\text { la prohibición de viaje. }\end{array}$ \\
\hline $\begin{array}{l}\text { Decreto Presidencial } \\
\text { sobre Principios } \\
\text { Fundamentales para } \\
\text { la Regulación del } \\
\text { Sistema Financiero de } \\
\text { los Estados Unidos del } \\
3 \text { de febrero de } 2017 .\end{array}$ & $\begin{array}{l}\text { Este decreto busca generar códigos eficientes y efectivos para racionalizar } \\
\text { el marco regulatorio financiero para el mercado nacional e internacional. } \\
\text { La administración Trump sostiene que esto permitiría a las empresas } \\
\text { norteamericanas ser más competitivas. Esta orden dirige al secretario } \\
\text { de Tesoro para que analice todas las leyes y códigos financieros actuales } \\
\text { con la intención de hacerlos más "eficientes". La vaguedad de estos } \\
\text { términos podría representar una amenaza para la continuidad de la Ley } \\
\text { Dodd-Frank, promulgada por Obama, para reducir el riesgo de sufrir } \\
\text { una nueva crisis del sistema financiero. }{ }^{4}\end{array}$ \\
\hline $\begin{array}{c}\text { Mejoras para el } \\
\text { Cumplimiento de la } \\
\text { Seguridad Fronteriza } \\
\text { y Migratoria del } 25 \text { de } \\
\text { enero de } 2017 .\end{array}$ & $\begin{array}{l}\text { Esta orden instruye al secretario de Seguridad Interna para comenzar la } \\
\text { planeación, diseño y construcción de un muro a lo largo de frontera entre } \\
\text { México y los Estados Unidos usando fondos del gobierno federal. De igual } \\
\text { forma, este decreto pretende construir nuevos centros de detención cerca de } \\
\text { la frontera, así como contratar a } 5000 \text { nuevos agentes de la patrulla fron- } \\
\text { teriza. Por otra parte, esta orden también busca cuantificar todo el apoyo } \\
\text { federal otorgado a México durante los últimos cinco años fiscales, aś como } \\
\text { empoderar a las policías locales para que tengan capacidades equivalentes } \\
\text { a oficiales de inmigración. El decreto ha sido fuertemente criticado por } \\
\text { diversas razones. Obtener el financiamiento necesario para la construcción } \\
\text { del muro ha resultado problemático, esto a pesar de los recortes presupues- } \\
\text { tales programados para otras agencias federales, tal como la Administración } \\
\text { de Seguridad del Transporte y la Guardia Costera. Durante su campaña, } \\
\text { Trump prometió que México pagaría el muro. Sin embargo, el gobierno } \\
\text { mexicano se ha negado de forma categórica ante esta solicitud del vecino } \\
\text { del norte. Por otra parte, el número de personas cruzando ilegalmente la } \\
\text { frontera ha disminuido en los últimos años. Finalmente, construir un muro } \\
\text { no confronta otro de los principales problemas de la inmigración ilegal, } \\
\text { el cual se relaciona con las personas que deciden permanecer en el país a } \\
\text { pesar de que su visa haya expirado. Todos estos factores contribuyen a la } \\
\text { falta de viabilidad de este decreto. }{ }^{5}\end{array}$ \\
\hline
\end{tabular}




\begin{tabular}{|c|c|}
\hline $\begin{array}{c}\text { Mejorando la } \\
\text { Seguridad Pública al } \\
\text { Interior de los Estados } \\
\text { Unidos del } 25 \text { de enero } \\
\text { de } 2017 .\end{array}$ & $\begin{array}{l}\text { Esta orden tiene como objetivo el agilizar la deportación de inmigrantes } \\
\text { ilegales en los Estados Unidos. Esto incluye la eliminación de ciudades } \\
\text { santuario, mismas que muestran tolerancia hacia dichos migrantes. Dicho } \\
\text { mandato busca bloquear toda adjudicación de fondos federales para } \\
\text { estas ciudades, así como contratar } 10000 \text { nuevos oficiales ejecutores } \\
\text { de deportación para el Departamento de Seguridad Interna (ICE). Ante } \\
\text { esta medida, los alcaldes de ciudades santuario como Nueva York, Los } \\
\text { Ángeles, Chicago o Boston han expresado que no cooperarán con el } \\
\text { gobierno federal para perseguir y criminalizar a los migrantes, dado que } \\
\text { los consideran miembros de sus comunidades. }{ }^{6}\end{array}$ \\
\hline $\begin{array}{c}\text { Decreto Ejecutivo } \\
\text { para la Expedición, } \\
\text { Revisión } \\
\text { Medioambiental y } \\
\text { la Aprobación de } \\
\text { Proyectos Prioritarios } \\
\text { de Infraestructura del } \\
24 \text { de enero de } 2017 .\end{array}$ & $\begin{array}{l}\text { Este decreto es parte de la estrategia del presidente Trump para } \\
\text { realizar megaproyectos de infraestructura durante su administración. } \\
\text { Esta instrucción dicta que el Consejo de Calidad Ambiental de la } \\
\text { Casa Blanca agilice los procedimientos y fechas de entrega de las } \\
\text { evaluaciones de impacto ambiental con el fin de agilizar los proyectos. } \\
\text { En esta misma fecha, el ejecutivo firmó otros decretos para autorizar } \\
\text { la construcción del oleoducto conocido como Keystone XL and Dakota } \\
\text { Access Oil Pipelines, proyecto que había sido terminantemente opuesto } \\
\text { por la anterior administración, así como un sinnúmero de activistas } \\
\text { ambientales. Durante su campaña, Trump prometió que el Gobierno } \\
\text { Federal obtendría la cuarta parte de las ganancias del proyecto, así } \\
\text { como el empleo de mano de obra americana y acero estadounidense } \\
\text { para la construcción del oleoducto. }\end{array}$ \\
\hline $\begin{array}{l}\text { Decreto Ejecutivo para } \\
\text { Minimizar la Carga } \\
\text { Económica de la } \\
\text { Derogación Pendiente } \\
\text { de la Ley de Protección } \\
\text { a los Pacientes y Salud } \\
\text { Asequible del } 20 \text { de } \\
\text { enero de } 2017 .\end{array}$ & $\begin{array}{l}\text { Este mandato permite al secretario de Salud y Servicios Humanos can- } \\
\text { celar o retrasar la implementación de las provisiones legales de la Ley } \\
\text { de Salud Asequible, mismas que pudieran generar una carga regulatoria } \\
\text { o financiera para cualquier estado o persona. Derogar y reemplazar la } \\
\text { ley antes mencionada, también conocida como Obamacare, es una de } \\
\text { las más prominentes promesas de campaña de Trump. No obstante, la } \\
\text { propuesta de salud pública del presidente } 45 \text { fue retirada del congreso } \\
\text { dado que no obtuvo el apoyo político necesario al interior del propio } \\
\text { Partido Republicano. }\end{array}$ \\
\hline
\end{tabular}

En la siguiente sección se detallan las escuelas de pensamiento utilizadas para el análisis de la presente investigación.

\section{ResPONSABILIDAD POLÍTICA, VOTO ECONÓMICO Y CONSTRUCCIÓN DE ESCENARIOS POLÍTICOS}

De acuerdo con la teoría de conexión electoral desarrollada por David Mayhew, ${ }^{16}$ la reelección representa un mecanismo que correlaciona la trayectoria de un político con los votantes. Teóricamente, si los funcionarios públicos actúan en contra de los intereses y prioridades de sus votantes, éstos los castigarán en las urnas, en conjunto con su partido.

\footnotetext{
${ }^{16}$ Mayhew, David, Congress: The Electoral Connection, New Haven, Yale University Press, 1974.
} 
Mayhew establece que los políticos se involucran en diferentes tipos de actividades para aumentar sus posibilidades de ganar una elección o una reelección. Esta dinámica crea una obligación y responsabilidad por parte de los funcionarios públicos a través de sus acciones y los resultados que éstas generan para el político y su partido en las elecciones subsecuentes.

Una de estas actividades es la publicidad (advertising), entendida como el esfuerzo de transmitir una imagen positiva del candidato, así como de su desempeño y capacidades. Una segunda actividad es el reclamo de crédito, lo cual promueve la creencia de que el candidato es personalmente responsable por implementar políticas exitosas y así lo expresa frente a los actores políticos relevantes. Esta última permite que los votantes perciban que el candidato es capaz de cumplir sus promesas y generar cambios importantes a través de políticas efectivas y eficaces. Mayhew propone que los funcionarios públicos electos tienden a diseñar actividades precisas para atraer audiencias y votantes específicos. De esta manera, un político adopta cierto tipo de causas y temas que el electorado considera importantes para lograr persuadir su intención de voto. ${ }^{17}$

El tercer tipo de acción realizada por los políticos es el posicionamiento, lo que implica realizar declaraciones públicas acerca de temas relevantes dentro de la agenda política. Los funcionarios electos expresan sus consideraciones y visión sobre los temas más importantes para la nación al tiempo que éstos ocurren. Asimismo, los políticos realizan propuestas para atacar los problemas sociales presentes en la agenda política y exponen de qué forma el público se beneficiaría de tomar dicho rumbo de acción en vez de otro. Este posicionamiento representa un riesgo político, específicamente en temas sustanciales para los votantes de su estado o distrito.

Tomar una posición resulta riesgoso por dos motivos: a) con el tiempo, la posición del político puede resultar equivocada, y b) los votantes que apoyen la posición contraria de inmediato tendrán un punto para rechazar al político. Por ello, tanto para políticos que buscan la reelección como para aquellos que tratan de cuidar la reputación su partido, es muchas veces preferible tomar posiciones mesuradas y apoyadas por la mayor parte de la sociedad. Actuar de forma altamente polémica y asumir una posición radical puede resultar desastroso para la reelección del político o para las aspiraciones de su partido. ${ }^{18}$

La opinión pública resulta clave para la representación, transparencia y rendición de cuentas, así como para la toma de decisiones dentro de la esfera política. De acuerdo con diversos autores, existe una correlación directa entre

\footnotetext{
${ }^{17}$ Ibidem.

${ }^{18}$ Carson, Jamie, \& Engstrom, Erik, "Assessing the Electoral Connection: Evidence from the Early United States", American Fournal of Political Science, vol. 49, núm. 4.
} 
la opinión pública y la forma en que las políticas son diseñadas e implementadas. La comprensión de la forma en que la opinión pública se transforma y cambia a través del tiempo es profundamente relevante para los políticos. Consecuentemente, es esencial desarrollar una alta especialidad y habilidades para evaluar estas dinámicas, mismas que permitirían a los políticos reelegirse o competir por otras posiciones.

De acuerdo con Zaller, ${ }^{19}$ en los Estados Unidos existe una correlación entre la opinión pública y el comportamiento electoral. Este autor argumenta que el público responde a la información proporcionada por las élites y tienden a formular sus opiniones tomando como base a los líderes de opinión en temas específicos. Las percepciones cambian a través del tiempo y los políticos deben ser conscientes y saber identificar y aprovechar dichas fluctuaciones para permanecer o competir por puestos de elección popular. ${ }^{20}$

Otra dimensión igualmente relevante se relaciona con el concepto de responsabilidad política. De acuerdo con Przeworski, Manin y Stokes, ${ }^{21}$ los votantes premian o castigan a los funcionarios electos gracias al éxito de sus políticas. En tal caso, los votantes tienden a votar de forma retrospectiva cuando enfrentan la opción de reelegir a sus representantes y así evalúan los efectos de las políticas implementadas por el político que detenta el poder, para removerlo o para que pueda conservar su puesto.

A pesar de que esto pudiera parecer intuitivo, diversos autores demuestran que los ciudadanos toman sus decisiones evaluando más los resultados de las políticas que los posicionamientos políticos del representante electo. Las posiciones políticas pueden llegar a cambiar conforme existan transformaciones en las condiciones sociopolíticas contextuales; sin embargo, la forma en que las políticas afectan a las personas es determinante para una decisión de voto. ${ }^{22}$

Otro tema altamente relevante dentro de la responsabilidad política tiene que ver con la erradicación del abuso de poder, así como del control en su uso. Como se mencionó en la primera sección, uno de los tipos de conducta que han generado juicios políticos de impugnación presidencial en los Estados Unidos ha sido precisamente el abuso de poder, específicamente en los casos de Nixon y Clinton. En este caso podemos observar que la dimensión de responsabilidad política es determinante para las reelecciones, dado que, en ambos casos, los partidos de Nixon y Clinton perdieron las elecciones subsecuentes. De acuerdo con Francis Fukuyama, la esencia de la responsabilidad

\footnotetext{
${ }^{19}$ Zaller, John, The Nature and Origins of Mass Opinion, Cambridge, Cambridge University Press, 1992.

${ }^{20}$ Ibidem.

${ }^{21}$ Przeworski, Adam, Manin, Bernard, \& Stokes, Susan, Democracy, Accountability, and Representation, New York, Cambridge University Press, 1999.

${ }^{22}$ Ibidem.
} 
política reside en la creencia de que los gobernantes se deben a los ciudadanos y que sus acciones deben estar dictadas por el bien común. ${ }^{23}$

De forma opuesta, si las personas perciben que los políticos persiguen únicamente su beneficio personal, es altamente probable que expresen su rechazo en las urnas en las siguientes elecciones. Esta expresión de responsabilidad política también evalúa la forma en que los gobiernos dan a conocer sus acciones en términos de transparencia. Este tipo de transparencia es vertical, dado que se demanda desde la sociedad civil, los ciudadanos y los medios. En caso contrario, cuando las agencias gubernamentales evalúan el desempeño de áreas específicas, se considera una transparencia horizontal.

Otro aspecto muy significativo de la responsabilidad política es la rendición de cuentas, la cual se refiere a la claridad y veracidad con la que los gobiernos publican su desempeño en cuenta pública, recaudación, presupuestos y políticas. Un elemento más lo constituye la aplicación de la ley (enforcement), así como de las sanciones en contra de funcionarios que hubieran abusado de su poder o violado el Estado de derecho. ${ }^{24}$

Existe una relación directa entre la existencia de estos mecanismos de rendición de cuentas y el nivel de confianza que éstos crean en el electorado. Ésta se traduce en una mayor participación política, tanto en elecciones, como en el desarrollo de organizaciones democráticas. ${ }^{25}$

La teoría del voto económico plantea una correlación directa entre el desempeño de la economía y la reelección de la administración en funciones. De acuerdo con numerosos estudios los votantes darán su voto aquellos políticos o partidos en el poder, en caso de que la economía tenga un desempeño positivo, inclusive si el crecimiento es marginalmente aceptable. En caso contrario, los votantes norteamericanos tienden a castigar al partido o político cuando la economía tiene un desempeño negativo.

Así, la percepción sobre el desempeño de la economía es la principal variable que determina la reelección de un político y de su partido. Asimismo, ha sido comprobado que los votantes deciden a quién otorgar el sufragio desarrollando un análisis retrospectivo; es decir, de acuerdo con la percepción "ex post" del desempeño económico de la administración en turno. ${ }^{26}$

La teoría del voto económico ha sido examinada y validada en Estados Unidos desde la década de los ochenta. ${ }^{27}$ Con ello se confirma que los

\footnotetext{
${ }^{23}$ Fukuyama, Francis, The origins of political order: from prehuman times to the French Revolution, New York, Farrar, Straus and Giroux, 2011.

${ }^{24}$ Schedler, Andreas, Diamond, Larry, \& Plattner, Mark, The Self-restraining State: Power and Accountability in New Democracies, Boulder, Lynne Rienner Publishers, 1999.

${ }^{25}$ Ríos, Alejandra, Cortés, Alejandro, Suárez, María, \& Fuentes, Laura, "Accountability: aproximación conceptual desde la filosofía política y la ciencia política”. Colombia Internacional, núm. 82.

${ }^{26}$ Lewis-Beck, Michael, \& Nadeau, Richard, op. cit.

${ }^{27}$ Kiewiet, Roderick, Macroeconomics and Micropolitics: The Electoral Effects of Economic Issues, Chicago, University of Chicago Press, 1983.
} 
votantes estadounidenses deciden su voto a partir de un análisis retrospectivo y sociotrópico del desempeño de la economía y de otros factores decisivos para cada sector electoral. Consecuentemente, diversos estudios han revelado que, si bien la economía es el principal criterio para otorgar el voto a uno u otro candidato, existen otros elementos respecto al elector que determinarán su voto.

Esto implica que temas como el gasto en seguridad social, la seguridad personal y familiar o la calidad educativa tienen un peso específico para seleccionar una opción política. La administración de Trump ha hecho diversos pronunciamientos y tomado acciones que han polarizado a la opinión pública, especialmente en temas de seguridad social, educación y, sobre todo, inmigración. Por lo tanto, estas acciones tendrán un costo político a corto y mediano plazo.

El uso de la vigésimo quinta enmienda para remover a Trump de la presidencia resultaría un hecho sin precedentes. Como ya se mencionó anteriormente, sólo se requiere que doce de los veintitrés miembros principales del gabinete y el vicepresidente decidan promover este recurso constitucional. ¿De qué forma podríamos evaluar la probabilidad de este escenario? Los modelos teóricos de prospectiva plantean la toma de decisiones bajo condiciones de incertidumbre.

Esta escuela basa sus corolarios en los conceptos de la teoría económica clásica sobre elección racional y economía conductual. No obstante, llega a suceder que los actores racionales no actúan de forma "racional". La teoría prospectiva modifica la elección racional; construye experimentos dentro de los cuales los participantes consistentemente actúan de maneras que violan los supuestos esenciales del modelo de elección racional. Los componentes esenciales de la teoría prospectiva son el encuadre (framing), ámbito (domain), aversión al riesgo (risk aversion), aversión a las pérdidas (loss aversion) y dotación (endowment).

Tversky y Kahneman consideran que la toma de decisiones depende primordialmente de la forma en que un problema es enmarcado. Dicho encuadre proporciona el contexto y contenido para analizar las posibles consecuencias de la elección de uno u otro rumbo de acción planteados para solucionar el problema. Asimismo, las opciones también dependen del ámbito o contexto para el cual son seleccionadas, mismas que a su vez surgen del encuadre.

Ambos conceptos guardan una relación simbiótica. Tversky y Kahneman sugieren que existen dos posibles ámbitos: pérdida o victoria. Si la elección es encuadrada desde una perspectiva de pérdida, las opciones son diferentes cuando ésta se plantea desde un escenario de victoria. De forma específica, la aversión al riesgo de las personas varía dependiendo del ámbito o contexto. $\mathrm{Al}$ contrario, bajo condiciones de ganancia o victoria, las mismas personas tienen una mayor aversión al riesgo. 
Finalmente, la dotación es el proceso mediante el cual las personas otorgan un mayor valor a las posesiones de lo que "racionalmente" harían. Por ejemplo, una persona que compra una taza de café por un dólar trataría de venderla por más dinero.

En este trabajo exponemos dos futuros realizables, considerando las posibles alteraciones que rodean tanto al presidente de los Estados Unidos, como al partido en el poder. Como podemos observar a partir de las teorías expuestas, existe una relación causa y efecto entre el desempeño gubernamental y el comportamiento político-electoral. Luego entonces, es posible prospectar que los efectos de las acciones, iniciativas legislativas y políticas del presidente Trump tendrán un efecto determinante en los próximos comicios de la Unión Americana.

El presidente Donald Trump ha asumido posiciones políticas altamente controversiales respecto a numerosos aspectos de políticas, así como de temas relevantes en la agenda pública. Como se expresó en la sección anterior, esto implica un alto riesgo respecto a los efectos que dichos posicionamientos pudieran tener dentro de un volátil contexto social y político. Tomando en cuenta las teorías anteriormente expresadas, presentamos dos posibles escenarios respecto al futuro de Trump. Cabe resaltar que no planteamos que los siguientes sean los únicos casos posibles; tomamos estos escenarios basándonos en el marco conceptual antes propuesto.

\section{RELACIÓN ENTRE ACEPTACIÓN PRESIDENCIAL Y CRECIMIENTO ECONÓMICO}

Con fundamento en la teoría de voto económico, consideramos que la presente administración podría conseguir la reelección en el 2020, en caso de que pueda mantener la tendencia positiva alcanzada por la administración de Barack Obama, esto aún a pesar de manifestar posiciones altamente controversiales en numerosos temas. Este sería el resultado mayormente esperado gracias a las proyecciones de crecimiento de la OCDE, ${ }^{28}$ el cual marca un crecimiento estable y marginalmente positivo durante los próximos tres años para Estados Unidos. Podemos expresar lo anterior a través del siguiente diagrama construido a partir de los casos y las teorías presentadas.

28 "United States - Economic forecast summary (June 2016)", OCDE. [Consulta: 2 de enero, 2018]. Dispo-
nible en: http://www oecd org/eco/outlook/united-states-economic-forecast-summary-june-2016.htm 
Tabla 2. Matriz de prospectiva de voto económico

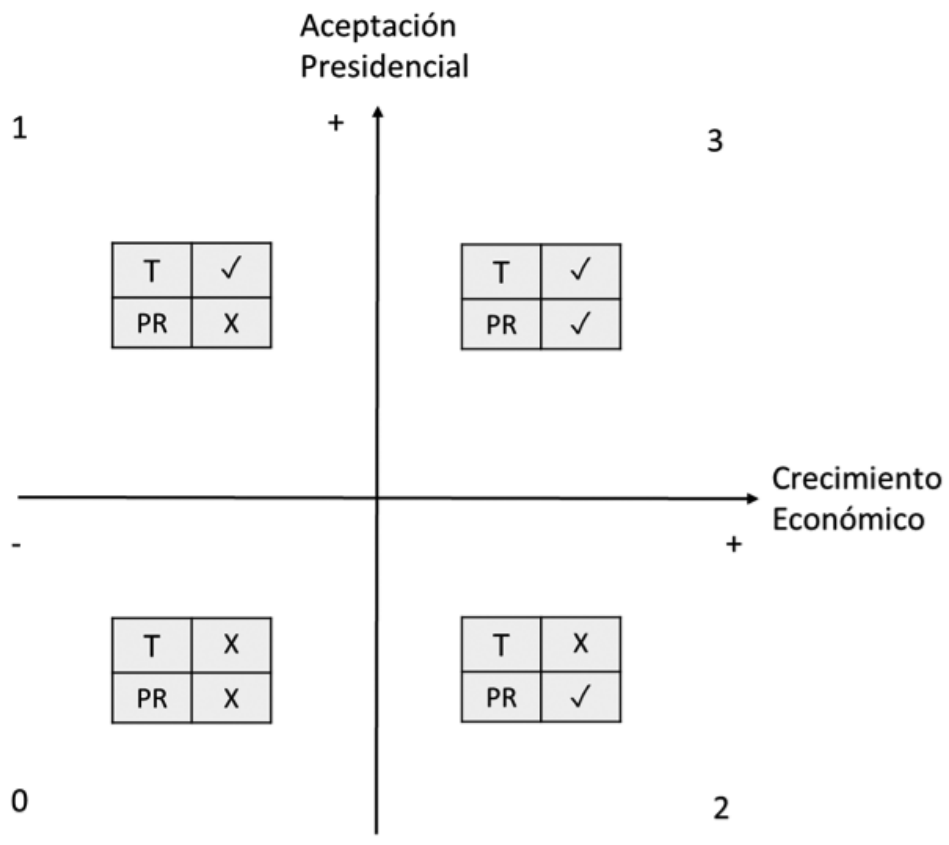

En este trabajo presentamos este método inédito para visulalizar la relación entre las teorías y los casos factibles. El cuadrante 3 representa una aceptación presidencial favorable y un crecimiento económico, lo cual asegura una reelección presidencial (T) en 2020 y una victoria del partido republicano (PR) en las elecciones intermedias del 2018. Este sería, considerando la teoría de voto económico, el caso más favorable para el GOP (Grand Old Party).

El cuadrante 2, con una baja aceptación presidencial y un positivo crecimiento económico, plantea una derrota en la reelección presidencial y una victoria parcial en las elecciones intermedias del 2018 para los republicanos. No sería posible asegurar si los republicanos mantendrían la mayoría en el Congreso, tal como ostentan actualmente. El cuadrante 1, donde la aceptación presidencial es alta pero el crecimiento es marginalmente positivo tendría una apretada victoria de Trump, pero también una derrota del Partido Republicano, dado que los votantes los castigarían en las elecciones intermedias por el pobre desempeño de la economía. No así la base electoral que apoya a Donald Trump, misma que le ha demostrado una gran fidelidad. Por último, en el cuadrante 0 , ante un desempeño negativo de la economía y un reducido margen de aprobación, tanto Trump como su partido tendrían derrotas en los próximos comicios por el Congreso y la Presidencia.

La siguiente gráfica muestra la aceptación popular del presidente Trump al transcurrir el primer año de su administración. Como dichos datos lo muestran, la popularidad de Trump ha disminuido considerablemente. Por 
ello, consideramos que su futuro político se encuentra íntimamente ligado con el desempeño que su administración tenga respecto al crecimiento económico, desempleo, comportamiento salarial y otros factores que impacten la percepción que el pueblo norteamericano tenga sobre el tema. Como conclusión de este escenario, planteamos que, gracias a la sinergia positiva de la economía, generada por la administración previa, es posible prever que Trump ganaría la reelección, a menos que suceda una catástrofe económica o su iniciativa de reforma fiscal genere consecuencias visiblemente negativas para los norteamericanos.

Tabla 3. Aceptación del presidente Trump

\begin{tabular}{|l|r|r|r|r|}
\hline \multicolumn{5}{|c|}{ Aceptación del presidente Trump } \\
\hline & \multicolumn{2}{|c|}{ ene-17 } & \multicolumn{2}{c|}{ ene-18 } \\
\cline { 2 - 5 } & Aprueba & Desaprueba & Aprueba & Desaprueba \\
\hline FiveThirtyEight & 45 & 45 & 37.6 & 57 \\
\hline Gallup & 45 & 47 & 38 & 58 \\
\hline IPSOS vía Reuters & 45.8 & 47.3 & 36.6 & 58.7 \\
\hline
\end{tabular}

Fuente: elaboración propia con datos de FiveThirtyEight, Gallup e Ipsos vía Reuters. Datos estadísticos recolectados en enero de 2018.

\section{RELACIÓN ENTRE ESTABILIDAD POLÍTICA Y DESEMPEÑO PRESIDENCIAL}

El siguiente diagrama relaciona la estabilidad política y el desempeño presidencial sustentado en la teoría de responsabilidad política. En el cuadrante tres, la percepción sobre el desempeño presidencial es positiva y existe estabilidad política, especialmente si la investigación conducida por Robert Mueller no tuviera ningún hallazgo significativo en cuanto a las acusaciones de posible colusión de la campaña presidencial y las autoridades del Gobierno ruso.

Esto incrementaría considerablemente la credibilidad y la aceptación del presidente Trump, sus posibilidades y las de su partido para ganar las elecciones de 2018 y 2020. El cuadrante dos tiene una baja o media estabilidad política y un positivo desempeño presidencial. Con ello, es posible anticipar una reelección presidencial de Trump, pero los republicanos sufrirían las consecuencias del enrarecido clima político, lo cual traería una derrota parcial para ellos en las próximas elecciones intermedias de 2018. 
El cuadrante uno plantea un resultado altamente negativo para Trump, en caso de que la investigación de Mueller tuviera hallazgos incriminatorios. En consecuencia, esto podría impulsar a los republicanos a invocar la XXV enmienda constitucional para removerlo del cargo, alegando una posible incapacidad física o mental para ejercer las funciones de su cargo, tal como se detalló anteriormente, solamente requiriendo la firma de la mayoría de los miembros del gabinete republicano. Otra opción es que el GOP use a Trump como un chivo expiatorio político al buscar recobrar la confianza de la ciudadanía estadounidense, retirándole el apoyo del partido. Esto le permitiría al GOP recuperar la confianza del público, al menos parcialmente, y les evitaría una derrota total en las elecciones por el control de la cámara y el senado en 2018.

El cuadrante 0 también considera una gran inestabilidad política, en conjunto con una decreciente popularidad por parte del mandatario, lo cual generaría derrotas para Trump y los republicanos. De acuerdo con la teoría de responsabilidad política y considerando tanto la decreciente popularidad del presidente norteamericano (véase tabla 3), así como los crecientes escándalos políticos y los altamente controversiales decretos presidenciales previamente detallados, es posible prever que Trump perdería las elecciones presidenciales del 2020. Pero será determinante que la investigación de Robert Mueller genere hallazgos profundamente negativos para el mandatario y su círculo íntimo. Solamente un hecho de esta naturaleza podría hacer que Trump pierda el apoyo de su base y de su partido.

Tabla 4. Matriz de prospectiva de responsabilidad política

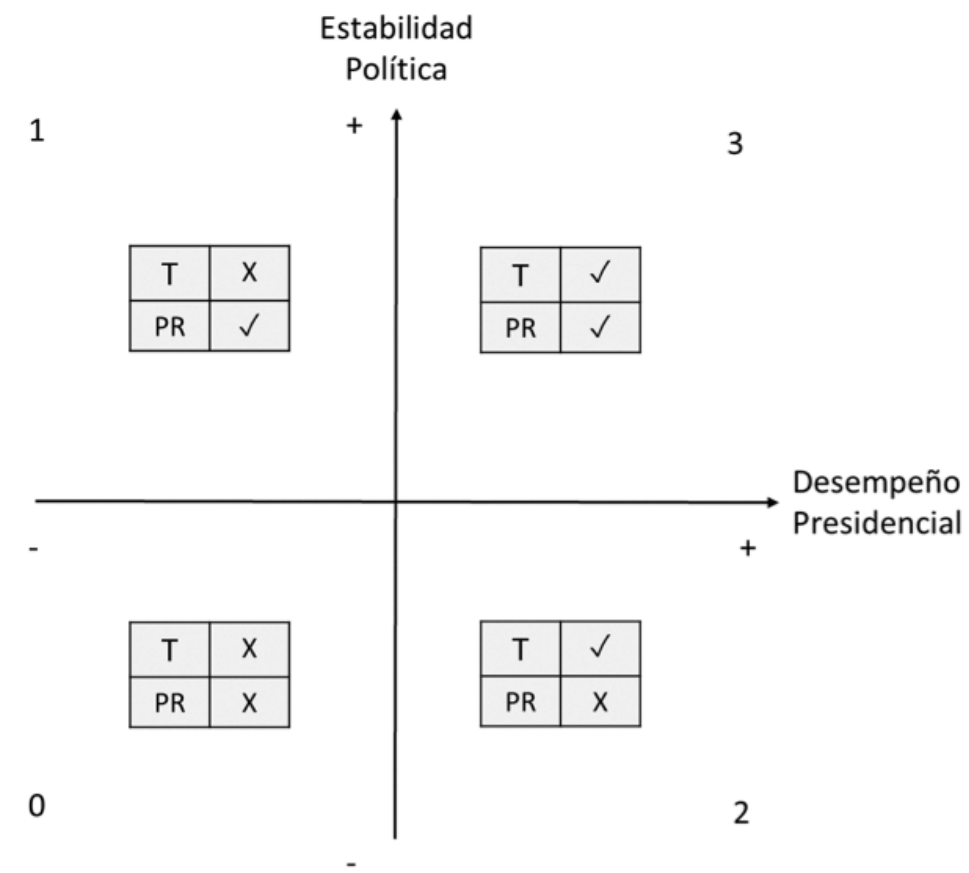


El nombramiento del fiscal especial Robert Mueller para conducir la investigación de cualquier tipo de vínculos o coordinación entre el Gobierno ruso y los individuos asociados con la campaña, así como cualquier tema que surja relacionado directamente con dicha investigación, demuestra un aumento de la presión política en contra de Trump.

Retomando las ideas antes expuestas sobre la teoría de prospectiva, es posible hacer un análisis sobre los posibles escenarios que podrían ocurrir con la administración Trump. Los incontables escándalos y una visible situación de caos al interior de la Casa Blanca proporcionan el encuadre para la presidencia de Trump. Considerando la teoría de elección racional antes expuesta, este encuadre sugiere que los republicanos están en un ámbito de pérdida (loss domain), dentro del cual los participantes son más propensos a aceptar el riesgo. Con ello, proponemos que se podría generar una mayor disposición por parte de los republicanos a tomar medidas para controlar y reducir los daños, respecto a los procesos electorales por el control del Congreso en 2018 y la presidencial del 2020.

En igual medida, es fundamental recordar que el gabinete presidencial está compuesto por personas con una fuerte lealtad personal hacia Trump. No obstante, figuras preponderantes del gabinete (como el vicepresidente Pence, el secretario del Trabajo Alexander Acosta, el secretario de Salud Tom Price, el secretario de Energía Rick Perry, así como la embajadora ante Naciones Unidas Nikki Haley, la secretaria de Transporte Elaine Chao, el director Mulvaney y el secretario de Vivienda Ben Carson) pudieran anteponer los intereses del partido republicano sobre su lealtad a Donald Trump, al ser políticos de carrera y con un fuerte arraigo republicano.

Otros miembros del gabinete, como los generales Mattis y Kelly, así como David Shulkin, secretario de Asuntos de Veteranos, podrían ser persuadidos de votar contra Trump por los riesgos que representa para la seguridad nacional. A pesar de todo lo anterior, resultaría difícil conseguir la mayoría de votos y firmas para proceder al uso de la XXV enmienda, a menos que la investigación Mueller tenga hallazgos determinantes.

\section{CONCLUSIONES}

Este artículo analiza dos posibles escenarios respecto a la presidencia de Donald Trump, con base en las consecuencias de sus propias declaraciones, decretos y políticas, tanto internacionales como domésticas. Una de estas posibilidades se basa en el vacío jurídico existente en la xxv enmienda. De acuerdo con la doctora Ellen Key, el contenido de esta enmienda es profundamente vago, ya que se usan términos como soborno, delitos y faltas graves, los cuales pueden significar muchas cosas, específicamente lo que el Congreso necesite para iniciar una acción legal en caso de así juzgarlo 
necesario. Siguiendo a Key, esta discrecionalidad en el sistema judicial se puede observar cuando los jueces federales tratan de establecer lo que significa "buen comportamiento". ${ }^{29}$

Finalmente, debemos mencionar una iniciativa surgida desde la sociedad civil y que muestra los alcances de las acciones de la actual administración. En octubre de 2017, se hizo pública la campaña "Need to Impeach", del multimillonario Tom Steyer, quien ha sido un gran donador del Partido Demócrata. La campaña busca recabar firmas para apoyar la impugnación de Trump y sugiere a los firmantes contactar directamente a sus representantes en el congreso para que se unan a esta iniciativa. ${ }^{30}$

Steyer ha aportado 20 millones de dólares para la difusión en medios. Este multimillonario ha sido entrevistado en un gran número de medios y su anuncio fue transmitido en múltiples plataformas, desde las pantallas de Times Square, hasta las pausas comerciales de la Serie Mundial, e inclusive en la cadena Fox News, a pesar de su expreso favoritismo por Trump. El anuncio acusa directamente a Donald Trump de tener a Estados Unidos al borde de una guerra nuclear con Corea del Norte, así como de obstruir la justicia y hacer uso indebido de fondos internacionales, por lo que es necesario impugnarlo. Hasta el miércoles 31 de enero de 2018, esta iniciativa ha logrado recabar más de 4.4 millones de firmas. ${ }^{31}$ Este hecho es solamente un punto más por considerar dentro del futuro político del cuadragésimo quinto presidente de los Estados Unidos.

\section{B I BLIOGRAF ÍA}

"2 Federal Judges Rule against Trump's Latest Travel Ban”, The New Tork Times. [Consulta: 28 de mayo, 2017]. Disponible en: https://www.nytimes. com/2017/03/15/us/politics/trump-travel-ban.html

"A Warning on Trump's Religious Liberty Order", The New York Times. [Consulta: 24 de mayo, 2017]. Disponible en: https://www.nytimes.com/2017/05/10/ opinion/trump-religious-liberty-order.html

"An Overview of the Impeachment Process", United States Senate. [Consulta: 16 de diciembre, 2017]. Disponible en: https://www.senate.gov/reference/resources/pdf/98-806.pdf

"Candidate Trump attacked Obama's executive orders. President Trump loves executive orders", The Washington Post. [Consulta: 10 de noviembre, 2017]. Disponible en: https://www.washingtonpost.com/news/monkey-cage/wp/2017/10/17/

\footnotetext{
${ }^{29}$ Entrevista con la doctora Ellen Key, 2017. La doctora Key, de la School of Justice and Government Studies de Appalachian State University, fue entrevistada por los autores el 18 de abril de 2017.

30 "Tom Steyer defends $\$ 20 \mathrm{M}$ ad campaign calling to impeach Trump", CNN. [Consulta: 25 de noviembre, 2017]. Disponible en: http://edition.cnn.com/2017/11/26/politics/tom-steyer-defends-impeachment-ads/index.html

31 "Need to Impeach Campaign", Need to impeach. [Consulta: 31 de enero, 2018]. Disponible en: https:// www.needtoimpeach.com/
} 
candidate-trump-attacked-obamas-executive-orders-president-trump-lovesexecutive-orders/?utm_term=.c12586dc46fb

Carson, Jamie, \& Engstrom, Erik, "Assessing the Electoral Connection: Evidence from the Early United States", American Fournal of Political Science, vol. 49, núm. 4.

Cely, Alexandra, "Metodología de los Escenarios para Estudios Prospectivos", Revista Ingeniería e Investigación, núm. 44.

Fukuyama, Francis, The origins of political order: from prehuman times to the French Revolution, New York, Farrar, Straus and Giroux, 2011.

Ginsberg, Benjamin, Lowi, Theodore., \& Weir, Margaret, We the People: An Introduction to American Politics, New York, W. W. Norton \& Company, 2014.

"Growing signs of trouble for Trump's border wall", Politico. [Consulta: 30 de mayo, 2017]. Disponible en: http://www.politico.com/story/2017/03/ trump-border-wall-politics-235904

"Impeachment and Removal", Congressional Research Service. [Consulta: 13 de enero, 2017]. Disponible en: https://fas.org/sgp/crs/misc/R44260.pdf

"Impeachment inquiry: William Jefferson Clinton, President of the United States". U.S. Government Publishing Office. [Consulta: 10 de enero, 2017]. Disponible en: https://www.gpo.gov/fdsys/pkg/GPO-CDOC-106sdoc3/pdf/GPO-CDOC106sdoc3-9.pdf

Kiewiet, Roderick, Macroeconomics and Micropolitics: The Electoral Effects of Economic Issues, Chicago, University of Chicago Press, 1983.

Lewis-Beck, Michael, \& Nadeau, Richard, "Economic voting theory: Testing new dimensions", Electoral Studies, vol. 30.

"List of presidents who were impeached", ThoughtCo, lifelong learning. [Consulta: 10 de enero, 2017]. Disponible en: https://www.thoughtco.com/ presidents-who-were-impeached-3368130

Mahmoud, Mohammed, Liu, Yuqiong, Hartmann, Holly, Stewart, Steven, Wagener, Thorsten, Semmens, Darius, et al., "A formal framework for scenario development in support of environmental decision-making", Environmental Modelling $\mathcal{E}$ Software, vol. 24, núm. 7.

Mayhew, David, Congress: The Electoral Connection, New Haven, Yale University Press, 1974.

Nardulli, Peter, Popular Efficacy in the Democratic Era: A Reexamination of Electoral Accountability in the United States, 1828-2000, Princeton, Princeton University Press, 2005.

"Need to Impeach Campaign", Need to impeach. [Consulta: 31 de enero, 2018]. Disponible en: https://www.needtoimpeach.com/

Przeworski, Adam, Manin, Bernard, \& Stokes, Susan, Democracy, Accountability, and Representation, New York, Cambridge University Press, 1999.

Ríos, Alejandra, Cortés, Alejandro, Suárez, María, \& Fuentes, Laura, "Accountability: aproximación conceptual desde la filosofía política y la ciencia política". Colombia Internacional, núm. 82.

"Sanctuary City' Mayors Vow to Defy Trump's Immigration Order", The New York Times. [Consulta: 1 de junio, 2017]. Disponible en: https://www.nytimes. com/2017/01/25/nyregion/outraged-mayors-vow-to-defy-trumps-immigration-order.html?_r=0

Schedler, Andreas, Diamond, Larry, \& Plattner, Mark, The Self-restraining State: Power and Accountability in New Democracies, Boulder, Lynne Rienner Publishers, 1999. 
"The Watergate Scandal", The Constitutional Rights Foundation. [Consulta: 11 de enero, 2017]. Disponible en: http://www.crf-usa.org/images/pdf/members/ bria25_4wb.pdf

“Tom Steyer defends $\$ 20 \mathrm{M}$ ad campaign calling to impeach Trump”, CNNN. [Consulta: 25 de noviembre, 2017]. Disponible en: http://edition.cnn.com/2017/11/26/ politics/tom-steyer-defends-impeachment-ads/index.html

"Trump has already signed 66 executive actions; here is what each one does", Business Insider. [Consulta: 25 de mayo, 2017]. Disponible en: http://www.businessinsider.com/trump-executive-orders-memorandum-proclamations-presidentialaction-guide-2017-1

"Trump is on pace to sign more executive orders than any president in the last 50 years", CNN. [Consulta: 29 de agosto, 2017]. Disponible en: http://edition. cnn.com/2017/10/13/politics/donald-trump-executive-orders/index.html

"Trump signed 90 executive actions in his first 100 days", Business Insider. [Consulta: 4 de mayo, 2017]. Disponible en: http://www.businessinsider.com/trumpexecutive-orders-memorandum-proclamations-presidential-action-guide-2017-1

"Trump signs executive actions to advance Keystone, Dakota Access pipelines", Politico. [Consulta: 1 de junio, 2017]. Disponible en: http://www.politico.com/ story/2017/01/trump-expected-to-sign-executive-actions-to-advance-keystonedakota-access-pipelines-234095

"Trump signs order to begin rolling back Wall Street regulations", The Washington Post. [Consulta: 28 de mayo, 2017]. Disponible en: https://www.washingtonpost. $\mathrm{com} /$ business/economy/trump-signs-order-to-begin-rolling-back-wall-streetregulations/2017/02/03/650668d8-ea30-11e6-80c2-30e57e57e05d_story. html?utm_term $=.00 f f a c 09 f 39 \mathrm{a}$

"Trump's art of no deal: Find someone to blame", CNN. [Consulta: 1 de junio, 2017]. Disponible en: http://edition.cnn.com/2017/03/24/politics/ donald-trump-health-care-blame/

"United States - Economic forecast summary (June 2016)", OCDE. [Consulta: 2 de enero, 2018]. Disponible en: http://www.oecd.org/eco/outlook/united-stateseconomic-forecast-summary-june-2016.htm

"What Trump's H-1B visa executive order actually does", Vox. [Consulta: 25 de mayo, 2017]. Disponible en: http://www.vox.com/ policy-and-politics/2017/4/18/15340686/hlb-visa-executive-order-trump

Zaller, John, The Nature and Origins of Mass Opinion, Cambridge, Cambridge University Press, 1992. 